\title{
Star Formation and Chemical Evolution of Lyman-Break Galaxies
}

\author{
Chenggang Shu \\ Shanghai Astronomical Observatory and National Astronomical \\ Observatories, Shanghai 200030, P.R.China
}

\begin{abstract}
The number density and clustering properties of Lymanbreak galaxies (LBGs) observed at redshift $z \sim 3$ are best explained by assuming that they are associated with the most massive haloes at $z \sim 3$ predicted in hierarchical models of structure formation. In this paper we study, under the same assumption, how star formation and chemical enrichment may have proceeded in the LBG population. A consistent model, in which the amount of cold gas available for star formation must be regulated, is suggested. It is found that gas cooling in dark haloes provides a natural regulation process. In this model, the star formation rate in an LBG host halo is roughly constant over about 1 Gyr. The predicted star formation rates and effective radii are consistent with observations. The metallicity of the gas associated with an LBG is roughly equal to the chemical yield, or about the order of $1 Z_{\odot}$ for a Salpeter IMF. The contribution to the total metals of LBGs is roughly consistent with that obtained from the observed cosmic star formation history. The model predicts a marked radial metallicity gradient in a galaxy, with the gas in the outer region having much lower metallicity. As a result, the metallicities for the damped Lyman-alpha absorption systems expected from the LBG population are low. Since LBG halos are filled with hot gas in this model, their contributions to the soft X-ray background and to the UV ionization background are calculated and discussed.
\end{abstract}

